\title{
12 Theorien des gestischen Sprachursprungs
}

It can be argued that [...] the origin of human sign languages is coincident with the origin of human languages; sign languages, that is, are likely to have been the first true languages.

Armstrong \& Wilcox (2007: 5)

\subsection{Der Kern gestischer Sprachursprungstheorien}

Mit Sicherheit liegt mit der in diesem Zitat skizzierten Position ein provokantes Szenario vor, den Ursprung von Sprachbefähigung zu erklären. „Given that we are such a talkative species, this may seem perverse. " heißt es in diesem Sinne nicht ohne Ironie bei Corballis (2002: Preface) gegenüber der Position eines gestischen Sprachursprungs - und dennoch finden sich eine Reihe von Autoren innerhalb der modernen Sprachursprungsforschung, welche diese Position mit variierender Stärke vertreten oder wenigstens in Erwägung ziehen und damit eine gemeinsame Tradition bilden (vgl. neben den soeben Erwähnten bspw. Armstrong, Stokoe \& Wilcox 1995, Donald 2005, Tomasello 2008 sowie Sterelny 2012).

Freilich wurde mit jenem oben angeführten Zitat eine besonders pointierte und einseitige Formulierung aus einem größeren Gesamtkontext gegriffen. Dagegen erkennen Armstrong und seine Kollegen durchaus an, dass Oralität in der gesamten Menschheitsentwicklung eine Rolle zu spielen hatte: Die Aussagen „At no time in our entire evolutionary history did communication take place in a single modality.“ (Armstrong \& Wilcox 2007: 37) sowie „Nor do we deny that hominids have made use of the vocal tract for social communication from the very beginning.“ (Armstrong, Stokoe \& Wilcox 1995: 26) zeugen hiervon. Daneben vertreten sie gewiss nicht die Position, dass mit Sicherheit vollwertige Gebärdensprachen entstanden seien, bevor die orale Domäne sprachlich erschlossen wurde, sondern konzentrieren sich vornehmlich auf die Aussage, dass die primäre und ursächliche Modalität innerhalb der Sprachevolution die gestische gewesen sei (bspw. Armstrong \& Wilcox 2007: 38 sowie 68).

Dabei findet die zentrale Auslegung der Datenlage beständig im Sinne ebenjenes Szenarios statt, dass eine sprachliche Befähigung sich evolutionär über die gestische Modalität entwickelt hätte und dass sprachliche Äußerungen auch heute noch im Kern als Gesten zu beschreiben seien: „We take the view that language is based in gesture - that is, bodily movement to which human beings attach meaning“ (Armstrong, Stokoe \& Wilcox 1995: 3). Deren Definition einer Geste umschließt dabei eben auch das Sprechen (bspw. Armstrong, 
Stokoe \& Wilcox 1995: 6): „The general model encompassing both spoken and signed languages to be presented here assumes that the key lies in describing both with a single vocabulary, the vocabulary of neuro-muscular activity - i.e. gesture.“ Dies führt zu einer konzeptuellen Auslegung, welche die höchst kritische Frage des Wechsels von der gestischen Modalität zum Sprechen zu umgehen sucht (Armstrong, Stokoe \& Wilcox: 42): „For us, the answer to the question, ,If language began as gesture, why did it not stay that way? is that it did.“ Dies beantwortet jedoch jene kritische Frage nicht, sondern verschiebt sie lediglich um eine Ebene, denn ganz im Sinne klassischer gestischer Sprachursprungstheorien halten sie daran fest, „[that] there is solid evidence in favor of visible gestures“ (Armstrong, Stokoe \& Wilcox: 58), wann immer die Ursprünge moderner Sprachbefähigung verortet werden sollen. Auch weitere Veröffentlichungen der Autorengruppe zeigen sich als klar in dieser Art zu klassifizieren (vgl. Armstrong \& Wilcox 2007: 64 sowie Armstrong 2008: 290 für unmissverständliche Feststellungen in diesem Sinne).

Gleiches oder Ähnliches gilt freilich auch für alle anderen zuvor genannten Autoren. Das Zentrum der diese einzelnen Autoren übergreifenden Position liegt trotz individueller Besonderheiten wiederholt an gleicher konzeptueller Stelle. Corballis (2002: Preface) schreibt „The main theme of the story is that language evolved, not from the vocal calls of our primate ancestors, but rather from their manual and facial gestures.“ Donald (2005: 293) stimmt prinzipiell überein, auch wenn er mit den Worten „[T]he first leap towards a distinctive hominid culture had to be a motoric one. Cultural expression took the form of whole-body action[.]“ mehr als Gesten in einem engeren Sinn umfasst. Tomasello (2008: 2) skizziert seine Position in ähnlicher Weise als „[M]y evolutionary hypothesis will be that the first uniquely human forms of communication were pointing and pantomiming.“ und Sterelny (2012: 2141) setzt die Tradition verteidigend fort: „This paper defends a gestural origins hypothesis about the evolution of enhanced communication and language in the hominin lineage.“

\subsection{Der historische Hintergrund gestischer Sprachursprungstheorien}

Ebendiese Tradition hat dabei tiefe Wurzeln. Gemeinhin wird Condillac als prototypischer Vertreter der modernen Position angesehen, indem er ab Mitte des 18. Jahrhunderts eine urförmliche „Aktionssprache“ vorschlug (vgl. Fischer 2011), auch wenn weniger explizite Ideenkonstrukte mit ähnlichen gedanklichen Neigungen bereits zuvor existierten (vgl. Fitch 2010: 438-439 für dessen 
Verweis auf Mandevilles Bienenfabel). Insbesondere zwei Feststellungen Condillacs zeigen dabei hohe Persistenz innerhalb der Geschichte der gestischen Sprachursprungstheorien. Einerseits vertrat er die Meinung, dass die Sprachleistung von Gebärdensprachen mit denen von oralen Sprachen vollumfänglich vergleichbar sind, und nimmt damit eine Position ein, welche sich in der breiteren Öffentlichkeit erst in der zweiten Hälfte des 20. Jahrhunderts durchsetzte. Andererseits konstatiert Condillac den Gehörlosensprachen eine innere Natürlichkeit und Eleganz, welche eine gestisch-pantomimische Ursprache bereits inhärent nahelegen soll. Auch wenn hier durchaus eine naive sowie unbegründete und damit geradezu unwissenschaftliche Feststellung vorzuliegen scheint, so zeigen sich auch wissenschaftlich stringentere moderne Sprachursprungstheorien als in Zustimmung mit dieser Interpretation.

Erstaunlich ist die Einmütigkeit jener zentralen, motivierenden Ideen innerhalb der einschlägigen Forschung. Bereits Kruse (1827) stützte sich auf die Willkürlichkeit und daher fehlende Eignung von Lauten als Zeichen für kommunikative Einheiten, um Condillacs Intuitionen zu verteidigen bzw. fortzuführen, und auch moderne Autoren stellen sich hier freimütig hinzu. Armstrong (2008: 289) hält in diesem Sinne zwei zentrale Annahmen als bleibende Charakteristika aller gestischen Sprachursprungstheorien fest: „Two common themes running through gestural theories of the origin of language are that iconic visible gesture is more natural than speech as a communication device and that it solves the problem of accounting for the origin of completely arbitrary signs." Auch Tomasello (2008: 9) stellt fest ,that the human use of pointing and pantomiming-as the successor to ape gestures after things became cooperativeare ,natural' in a way that ,arbitrary' linguistic conventions are not. " und andere bringen Gleiches vor und beziehen sich dabei mitunter direkt auf Condillac (bspw. Corballis 2002: 64). Die Fortführung dieser Intuitionen darf als primäre Motivation für die Position gesehen werden, während einige der zugunsten der Position vorgebrachten Argumente nicht als motivierend, sondern vielmehr als eine ohnehin bereits bestehende Intuition bestärkend anzusehen sind. Dies geschieht dabei, wie an früherer Stelle bspw. anhand der Forschungsgeschichte des FOXP2-Gens dargestellt wurde, in einer oftmals durchaus problematischen Weise.

\subsection{Die Inhomogenität gestischer Sprachursprungstheorien}

Auf Basis dieser anscheinend sehr einheitlichen Tradition einschließlich einiger gleichförmiger zentralen Annahmen werden die gestischen Sprachursprungstheorien gemeinhin unter einem gemeinsamen Deckmantel zusammengefasst 
und als geschlossene Einheit angesehen. Die große Zahl einschlägiger Autoren aus verschiedenen Disziplinen, welche sich hier zusammenfassen lassen, suggeriert dabei eine großflächige und gut fundierte Gesamtposition. Bei genauerer Betrachtung zeigen sich diese gemeinsame Klassifikation sowie die sich daran anschließenden Werturteile jedoch als verfälschend. Dies offenbart sich zum einen bereits im Kontext einer näheren Untersuchung der einzelwissenschaftlich verorteten Argumente. Wie beispielhaft in mehreren Unterkapiteln anhand konkreten Beispielen erörtert wurde, zeigen sich einschlägige Argumentationslinien bei näherer Betrachtung oftmals als einseitig, idealisiert, übergeneralisiert sowie spekulativ, sodass die übergreifende Position als weniger gut fundiert zu gelten hat als es anhand der vorgebrachten Argumente den Anschein hat. In der vorliegenden Arbeit wurde derlei insbesondere innerhalb der Genetik zum FOXP2-Gen sowie in der Neurologie zu Spiegelneuronen dargelegt, jedoch wurden auch bei der Bewertung nicht-menschlicher Kommunikation sowie im Bereich der Paläoanthropologie Daten vorgebracht, welche einschlägigen Auslegungen widersprechen - ohne dass in dieser Hinsicht in der vorliegenden Arbeit Raum gegeben werden konnte.

Weiterhin und mit desaströser Folge für die involvierten Theoriekonstrukte zeigen sich die Autoren in ihren Vorgehensweisen und Schlussfolgerungen jenseits der bereits vorgestellten Grundannahmen als in einer Weise uneinheitlich, sodass anzuzweifeln ist, ob die Autoren rechtens unter einem gemeinsamen Deckmantel zusammengefasst werden können. Als ein Beispiel soll die zeitliche Verortung eines Sprachursprungs dienen. Corballis (2002: 108) stellt in dieser Hinsicht fest, dass eine Sprachbefähigung in Form von Gebärdensprache ein sehr altes Phänomen darstellen könnte: „[A] group of hominins, perhaps a million years ago, may well have achieved a form of language with the full grammatical range and expressiveness of the modern signes languages of the deaf[.]“ Darüber hinaus könnten protosprachliche Stufen einschließlich erster protosyntaktischer Strukturen bereits zu Beginn der Gattung des Menschen aufgekommen sein (Corballis 2002: 183): „The appearance of the larger-brained genus Homo some 2 million years ago may have signaled the emergence and later development of syntax[.]“ Eine Oralität der Sprache sei dagegen ein auf den anatomisch modernen Menschen beschränktes Phänomen und damit auf einen Zeitraum zwischen vor 200.000 und 50.000 Jahren zu verorten (Corballis 2002: 183 sowie 198$)$.

Tomasello (2008) spricht sich im Gegensatz hierzu gegen jegliche Sprachbefähigung außerhalb des anatomisch modernen Menschen aus. In der Tat kämen pantomimische Vorstufen zu Sprache erst im frühen H. sapiens auf und erst im späten $H$. sapiens bildeten sich sowohl eine vollwertige Sprachbefähigung als 
auch Oralität aus (vgl. zu all dem die zusammenfassende Abbildung in Tomasello 2008: 239). In anderen Worten kommen die beiden Autoren zu in größtem Maße abweichenden Schlussfolgerungen - ein Bild, das sich auch im Rahmen anderer Teilfragen ergibt, sodass sich hinsichtlich verschiedener Teilpositionen nennenswerte Gegensätze zwischen den Autoren einstellen. Damit stellt der Versuch, diese beiden Sprachursprungstheorien als sich gegenseitig stützend aufzufassen, ein äußerst zweifelhaftes Vorgehen dar.

Jenseits dieser Problematik abweichender Schlussfolgerungen bzw. Teilpositionen zeigt sich die Forschungslandschaft der gestischen Sprachursprungstheorien als feingliedriger als oftmals angenommen wird. Arbib (2012: Preface) schreibt: „Protosign and protospeech [...] evolved in an expanding spiral to yield the neural circuitry and social structures that enabled modern language to emerge.“ Dem und ähnlichen Aussagen ist zu folgern, dass Arbib (2012) nicht als ein Vertreter eines gestischen Sprachursprungs nach jener vormals skizzierten Tradition zu sehen ist, denn Oralität spielte laut Arbib (2012) in einem multimodalen, opportunistisch zweigleisigem System stets eine bedeutende Rolle, während jene anderen Autoren den treibenden Faktor sowie erste kommunikative Systeme fest in der gestischen Modalität verankern und Oralität als begleitendes Beiwerk charakterisieren. Da jedoch frühere Veröffentlichungen Arbibs dazu tendieren, die Bedeutung der gestischen Modalität stärker zu betonen als im vorgebrachten Zitat gegeben ist (vgl. dazu bspw. Rizzolatti \& Arbib 1998: 192), tendieren andere Autoren und insbesondere Vertreter eines gestischen Sprachursprungs dazu, Arbib als einen Vertreter der gestischen Tradition $z u$ klassifizieren (vgl. bspw. Armstrong 2008: 302).

Dies führt zu einer Situation, in welcher Autoren, die vollwertige Gebärdensprachen ohne nennenswerte Oralität für möglich halten (vgl. nochmals jenes das vorliegende Unterkapitel einleitende Zitat von Armstrong \& Wilcox 2007: 5), mit anderen, welche sich für eine rein protosprachliche gestische Phase aussprechen (siehe wiederum Tomasello 2008) oder welche einen multimodalen Ansatz verfolgen (wie soeben dargestellt bspw. Arbib 2012), unter dem gemeinsamen Deckmantel gestischer Sprachursprungstheorien zusammengefasst werden. Zugleich sind Autoren, welche sich in einer mit Arbib (2012) grob vergleichbaren Sphäre befinden - in der Hinsicht, dass sie eine multimodale Evolution von Sprachbefähigung als wahrscheinlich halten - von einer solchen Zusammenfassung durch die Ansichten der einschlägigen Literatur ausgenommen (vgl. als ein solches Beispiel die Position Bickertons 2003: 80-81 und 2007: 512). 


\subsection{Ein allgemeines Urteil zu gestischen (und weiteren) Sprachursprungstheorien}

Es erscheint damit als fahrlässig, von einem geschlossenen, d.h. konzeptuell einheitlichen, Feld gestischer Sprachursprungstheorien auszugehen. Die vorangegangenen Erläuterungen zusammenfassend ergibt sich dies erstens schon daraus, dass die Auslegung der gestischen Phase bei unterschiedlichen Autoren in sehr voneinander abweichender Weise geschieht. Zweitens verhärtet sich dieses Urteil unter Berücksichtigung der Tatsache, dass andere Teilfragen des Forschungsfeldes von ebenjenen Autoren unterschiedlich beantwortet werden, sodass sich deren Gesamtpositionen oft allein hinsichtlich der Konstatierung einer gestischen Phase berühren. Zuletzt schwächt sich die Stärke der Theorie bzw. Hypothese eines gestischen Sprachursprungs durch eine Reihe von übermäßig idealisierten Argumenten, welche von einschlägigen Autoren zugunsten der Position vorgebracht werden und welche im Licht einer genaueren Betrachtung respektive Rezeption der relevanten Ergebnisse der Einzelwissenschaften ganz oder teilweise relativiert werden müssen.

Es verbleibt festzustellen, dass sich ein ähnliches Bild auch zu Autoren zeigt, welche ihren jeweiligen Fokus auf andere Teilfragen bzw. Teilpositionen des einschlägigen Forschungsfeldes legen. Veröffentlichungen, welche sich für eine musische oder semi-musische Phase in der Sprachevolution aussprechen (vgl. Mithen 2005, Knight \& Lewis 2017 sowie potenziell auch Dunbar 1996 und Falk 2009), stehen in einem ähnlichen Verhältnis zueinander, und wieder andere Autoren orientieren sich ebenfalls anders hinsichtlich im vorhergehenden Kapitel zum Inventar der Sprachursprungsforschung vorgestellter Forschungsfragen bzw. Positionen zur Beantwortung dieser Forschungsfragen. Damit repräsentiert das Forschungsfeld als Ganzes ein konzeptuell unausgereiftes und problematisches wissenschaftliches Programm.

Eine stringente interdisziplinäre Zusammenarbeit der involvierten Einzelwissenschaften und eine leistungsfähige Synthese der einzelwissenschaftlichen Ergebnisse wird vonnöten sein, um zu näherungsweise allgemeingültigen Antworten kommen zu können. Ein derartiger Austausch hat dabei zur Voraussetzung, dass die involvierten Autoren ihre jeweilige Expertise gegenseitig anerkennen, angemessene Sensibilitäten für die Terminologie sowie Konzeptualisierung der jeweils anderen Disziplinen ausbilden und ein fachliches Verständnis wenigstens in einem Maße erarbeiten, sodass gröbste Fehler und Missverständnisse ausgeschlossen werden können, wenn am gemeinsamen Diskurs zur Forschungsfrage des Sprachursprungs teilgenommen wird. 
Entgegen dieser Anforderungen ist das Forschungsfeld umfänglich von einer Mentalität durchzogen, welche als konzeptuelles und terminologisches Chaos bezeichnet werden kann, denn derselbe Begriff bezeichnet bei unterschiedlichen Autoren stark abweichende Phänomene. Dies beruht unter anderem auf einer Missachtung ebenjener Prinzipien, welche soeben dargestellt wurden. Eine Verdeutlichung der daraus entstehenden defizitären Vorgehensweise geschah an vorangegangener Stelle unter anderem anhand linguistischer Begriffe wie der Holophrase und der Syntax sowie anhand biologischer Konzepte innerhalb der Genetik. In beiden Fällen geschah eine Verzerrung bzw. Zweckentfremdung der Konzepte und Begriffe zugunsten der jeweils eigenen Position, ohne dass damit auch nur prinzipiell ein gemeinsamer Diskurs aufgemacht werden könnte, denn für einen ebensolchen fehlt ein gemeinsames Vokabular. Das nachfolgende Kapitel versucht sich in einer weiterführenden Erschließung einschlägiger konzeptueller Probleme, um den vorliegenden Gedanken inklusive der damit zusammenhängenden Mahnung zu einer natürlichen Kulmination bringen zu können.

Im Sinne des dem vorliegenden Programm unterliegenden Methodenkatalogs soll zuvor noch knapp darauf verwiesen werden, dass zwar einige Prinzipien wie M1, M3 und M7 keinen besonderen Mehrwert für eine Bewertung der gegebenen Situation zu bringen scheinen, jedoch triangulieren M2 und M4 das soeben diagnostizierte Vorgehen der Übergeneralisierung - d. h. wenn beachtet, so hätten diese Prinzipien einschlägig vorhandenen Problemen vorbeugen können. Gleiches gilt für M5 sowie M9, welche beide vor einer verfrühten Feststellung von potenziell problematischen Schlussfolgerungen warnen. M8 schließlich stellt ein Prinzip dar, welches im weiteren Forschungsverlauf die fehlende Adäquatheit jener Schlussfolgerungen zunehmend $\mathrm{zu}$ offenbaren vermag, und M10 stellt einen simplen Test dar, welcher zeigt, dass die Gesamtsituation nicht in dem Maße klar zu sein scheint als einige Autoren darzustellen suchen. Zuletzt stellen M11 bis M13 Anreize, die Position eines gestischen Sprachursprungs sowohl in empirischer als auch in konzeptueller Weise zu hinterfragen - mit dem Ergebnis der Feststellung, dass das Feld der gestischen Sprachursprungstheorien mit nennenswerten Problemen zu kämpfen hat. Wie zuvor bereits angemerkt lassen sich diese Urteile auch jenseits gestischer Sprachursprungstheorien auf das Forschungsfeld der Sprachursprungsforschung anwenden. 